\title{
Smartphone applications to reduce alcohol consumption and help patients with alcohol use disorder: a state-of-the-art review
}

This article was published in the following Dove Press journal:

Advanced Health Care Technologies

II December 2015

Number of times this article has been viewed

\author{
Steven E Meredith \\ Sheila M Alessi \\ Nancy M Petry \\ Calhoun Cardiology Center, \\ University of Connecticut School of \\ Medicine, Farmington, CT, USA
}

\begin{abstract}
Hazardous drinking and alcohol use disorder (AUD) are substantial contributors to USA and global morbidity and mortality. Patient self-management and continuing care are needed to combat these public health threats. However, services are rarely provided to patients outside of clinic settings or following brief intervention. Smartphone applications ("apps") may help narrow the divide between traditional health care and patient needs. The purpose of this review is to identify and summarize smartphone apps to reduce alcohol consumption or treat AUD that have been evaluated for feasibility, acceptability, and/or efficacy. We searched two research databases for peer-reviewed journal articles published in English that evaluated smartphone apps to decrease alcohol consumption or treat AUD. We identified six apps. Two of these apps (A-CHESS and LBMI-A) promoted self-reported reductions in alcohol use, two (Promillekoll and PartyPlanner) failed to promote self-reported reductions in alcohol use, and two (HealthCall-S and Chimpshop) require further evaluation and testing before any conclusions regarding efficacy can be made. In summary, few evaluations of smartphone apps to reduce alcohol consumption or treat AUD have been reported in the scientific literature. Although advances in smartphone technology hold promise for disseminating interventions among hazardous drinkers and individuals with AUD, more systematic evaluations are necessary to ensure that smartphone apps are clinically useful.
\end{abstract}

Keywords: smartphone, app, mHealth, alcohol, alcohol use disorder, hazardous drinking

\section{Introduction}

Alcohol use is a leading cause of preventable morbidity and mortality in the USA and around the world. In 2012, 3.3 million deaths were attributable to alcohol consumption, $\sim 6 \%$ of deaths worldwide. ${ }^{1}$ Many alcohol-related deaths result from the overconsumption of alcohol due to hazardous drinking and alcohol-related mental health disorders, including alcohol use disorder (AUD), a problematic pattern of alcohol consumption leading to clinically meaningful impairment or distress. ${ }^{2}$ AUD is a common mental health disorder with recent estimates indicating a lifetime prevalence rate of $29.1 \%$ in the USA. ${ }^{3}$ Like other substance use and addictive disorders, AUD has been characterized as a chronic, relapsing disease. Thus, continuing care is critical to effective treatment. However, few services are provided to patients outside clinics or after acute intervention. ${ }^{4}$ Alcohol misuse is also problematic for individuals who engage in hazardous drinking (eg, binge drinking) but do not meet diagnostic criteria for alcohol-related disorders. For these individuals, brief intervention can promote reductions in alcohol consumption. ${ }^{5}$ Typically, however, such intervention only benefits those who access the health care system. For hazardous drinkers and individuals with
Correspondence: Nancy M Petry Calhoun Cardiology Center, University of Connecticut School of Medicine, 263 Farmington Avenue, Farmington, CT 06030, USA

$\mathrm{Tel}+\mathrm{I} 8606792593$

Fax +18606791312

Email npetry@uchc.edu 
AUD, mobile health (mHealth) technology may help bridge the gap between traditional care and clinical need.

Approximately half of the world's population, 3.6 billion people, were identified as mobile phone subscribers in $2014 .^{6}$ In the USA, $>90 \%$ of adults own mobile phones, with similar or greater rates of ownership among those from lower income households, individuals with no college education, those living in rural communities, and members of racial and ethnic minorities. ${ }^{7}$ In recent years, behavioral health researchers have developed and tested a variety of mobilephone-based mHealth interventions to treat various health conditions, including AUD. ${ }^{8}$ Many of these interventions rely on one of the most basic functions of mobile phones: text messaging. For example, Agyapong et $\mathrm{al}^{9}$ developed a textmessage-based intervention to improve mood and alcohol abstinence among patients with depression and comorbid AUD. Compared to participants in a control group, participants who received twice-daily supportive text messages for 3 months demonstrated significantly better mood and a nonsignificant trend toward greater rates of alcohol abstinence; however, these effects were no longer evident at a 6-month follow-up. ${ }^{10}$ Overall, text-message-based interventions have shown mixed results. ${ }^{8,11}$

More innovative and engaging mHealth interventions may be more efficacious. For example, Alessi and Petry ${ }^{12}$ developed and tested an incentive-based mHealth intervention to promote abstinence from alcohol in adults who drank frequently but were not seeking alcohol treatment services. Participants received a mobile phone and a breathalyzer and were instructed to submit video recordings of alcohol breath tests in response to researcher prompts that were sent daily over the course of a 4-week intervention. Participants were randomly assigned to one of two groups: 1) a control group $(n=15)$, in which participants received modest compensation for each video submitted, and 2) an abstinence-contingent group ( $\mathrm{n}=14$ ), in which participants received additional compensation contingent upon videos of breath samples indicative of abstinence. Participants assigned to the abstinence-contingent group demonstrated significantly longer durations of abstinence ( $M=16.8$ days) than participants assigned to the control group ( $M=5.9$ days, $P<0.001)$. Although the study sample was small, the results were robust.

mHealth interventions that rely on some of the more basic functions of mobile phones such as text-messaging and video recording are advantageous because they allow real-time monitoring in the natural environment. Interventions that harness the more sophisticated technological capabilities of smartphones (ie, advanced operating system, interactive touchpad screen, Internet connectivity, motion sensors, global positioning system [GPS], etc) may have additional advantages by providing intervention precisely when patients are at the highest risk of lapse. Thus, an increasing number of behavioral health researchers are working to develop smartphone-based mHealth software applications (“apps”).

An estimated $64 \%$ of US adults own smartphones, and among ethnic and racial minorities, smartphone ownership is even higher (eg, 70\% among Black, non-Hispanic and 71\% among Hispanic). In addition, 50\% of individuals who earn $<\$ 30,000$ per year currently own smartphones, and this percentage is increasing as more individuals with lower income are relying solely on smartphones for Internet access..$^{13}$ Thus, if current market trends continue, smartphone-based mHealth technology will soon be applicable to nearly all patients, regardless of background. Moreover, this technology will bring treatment to individuals who are not accessing the health care system due to a variety of barriers, including a desire to avoid social stigma associated with AUD treatment.

A recent review of commercially available apps ${ }^{14}$ identified 662 alcohol-related apps at the iTunes (iPhone) and Google Play (Android) stores. Although the majority of these apps were designed for entertainment purposes and actually promoted alcohol use, 91 of them were classified as alcohol reduction apps. Notably, however, even those apps that were designed to reduce alcohol consumption had little or no evidence base. Thus, much more research is needed on the design, development, and evaluation of evidence-based smartphone apps to treat AUD and reduce alcohol consumption. The purpose of the current review is to identify and discuss AUD and alcohol reduction smartphone apps that have been evaluated in the peer-reviewed scientific literature.

\section{Search methods and results}

We conducted searches of the titles and abstracts of publications indexed in two research databases (PubMed and Scopus) on July 1, 2015, using the following three combinations of search terms: "smartphone" and "alcohol", "mHealth" and "alcohol", and "eHealth" and "alcohol". After eliminating duplicates, 87 results were obtained. Studies selected for inclusion were peer-reviewed journal articles published in English that evaluated smartphone apps to decrease alcohol consumption or treat AUD. Reference sections of review papers and studies that met inclusion criteria were also searched. Published protocols of proposed clinical trials and technical reports of smartphone app development were excluded. Studies that examined only computer-based or tablet-based interventions were also excluded. 
Although many systematic reviews of behavioral health interventions include only interventions that were evaluated using randomized controlled clinical trials, the inclusion criteria for the current review were less stringent given that the topic of this review is still a relatively new field of study. Thus, we included any smartphone apps that were evaluated for feasibility, acceptability, and/or efficacy, through either preliminary testing (eg, pilot studies) or more rigorous empirical investigation (eg, randomized controlled trials). We identified six such smartphone apps. The apps and the relevant literature are listed in Table 1, and each app is summarized in the following narrative review.

\section{Alcohol - Comprehensive Health Enhancement Support System}

Alcohol - Comprehensive Health Enhancement Support System (A-CHESS) had the strongest evidence base of any of the smartphone apps for AUD that were identified in our search. ${ }^{15-19}$ A-CHESS is a relapse prevention app for individuals in recovery for alcohol dependence who have been recently discharged from residential care. The app is based on CHESS, a computerized system developed at the University of Wisconsin-Madison that has been used in the management of various diseases, including cancer, ${ }^{20,21}$ asthma, ${ }^{22}$ and $\mathrm{HIV}_{.}{ }^{23}$

A-CHESS is informed by self-determination theory, which suggests that motivation and well-being are enhanced when three psychological needs are met: competence, autonomy, and relatedness. ${ }^{24} \mathrm{It}$ is also informed by a cognitive-behavioral approach to relapse prevention that focuses on identifying and preventing high-risk relapse situations. ${ }^{25} \mathrm{~A}-\mathrm{CHESS}$ provides a variety of features and services that are consistent with this theoretical framework. For example, the app uses GPSenabled location tracking to alert users when they approach high-risk locations (eg, a bar that the patient once frequented). The app also offers information services via electronic libraries, the web, and an "ask-an-expert" feature. It provides social support via anonymous discussion forums, a text-messaging feature for preapproved friends and family, and a panic button that, if pressed, will contact the user's preapproved friends or family when he or she is in greatest need of support. It also provides text and audio reminders of milestones and reasons for quitting. In addition, it utilizes regular surveys to assess alcohol use, craving, and withdrawal symptoms, and it provides users with various relaxation tools.

In a recent randomized controlled trial, 349 patients completing residential care who met Diagnostic and Statistical Manual of Mental Disorders-IV diagnostic criteria for alcohol dependence were assigned to an 8-month treatment-as-usual control condition or a treatment-as-usual plus A-CHESS condition. ${ }^{18}$ Nearly $94 \%$ of participants who received A-CHESS accessed the app during the first week of the intervention. By the end of month $4, \sim 80 \%$ of A-CHESS participants were still using

Table I Smartphone apps to reduce alcohol consumption or treat AUD

\begin{tabular}{|c|c|c|c|}
\hline Name & Overview & References & Evidence of efficacy \\
\hline A-CHESS & $\begin{array}{l}\text { Supports AUD recovery with high-risk location tracking, } \\
\text { educational resources, social support, a panic button, } \\
\text { regular assessments, and relaxation tools }\end{array}$ & $15-19,26$ & $\begin{array}{l}\text { In a randomized controlled trial, participants } \\
\text { who received A-CHESS plus treatment as usual } \\
\text { reported significantly fewer risky drinking days than } \\
\text { participants who received only treatment as usual }{ }^{18}\end{array}$ \\
\hline LBMI-A & $\begin{array}{l}\text { Supports AUD recovery through a series of } \\
\text { psychoeducational modules and other features, including } \\
\text { high-risk location tracking, social support, regular } \\
\text { assessments, and motivational tools }\end{array}$ & $27-31$ & $\begin{array}{l}\text { In a pilot study, participants who received LBMI-A } \\
\text { reported significant decreases in self-reported } \\
\text { heavy drinking days and drinks per week and } \\
\text { a significant increase in the percentage of days } \\
\text { abstinent relative to baseline }{ }^{31}\end{array}$ \\
\hline Promillekoll & $\begin{array}{l}\text { Swedish-language app that estimates BAC based on user } \\
\text { input regarding quantity and quality of alcoholic beverages }\end{array}$ & 36 & $\begin{array}{l}\text { In a randomized controlled trial, participants } \\
\text { who received Promillekoll reported no significant } \\
\text { decreases in alcohol consumption relative to a no- } \\
\text { intervention control }{ }^{36}\end{array}$ \\
\hline PartyPlanner & $\begin{array}{l}\text { Swedish-language app that estimates BAC based on user } \\
\text { input regarding quantity and quality of alcoholic beverages } \\
\text { and allows users to simulate drinking occasions before } \\
\text { they occur to predict and compare BAC estimates }\end{array}$ & 36 & $\begin{array}{l}\text { In a randomized controlled trial, participants who } \\
\text { received PartyPlanner reported no significant } \\
\text { decreases in alcohol consumption relative to a no- } \\
\text { intervention control }{ }^{36}\end{array}$ \\
\hline HealthCall-S & $\begin{array}{l}\text { Facilitates motivational interviewing via assessment and } \\
\text { feedback of alcohol consumption patterns }\end{array}$ & 39 & $\begin{array}{l}\text { Limitations of preliminary investigations preclude } \\
\text { any definitive statements regarding app efficacy }{ }^{39}\end{array}$ \\
\hline Chimpshop & Trains users to overcome alcohol attentional bias & 40,41 & $\begin{array}{l}\text { Limitations of preliminary investigations preclude } \\
\text { any definitive statements regarding app efficacy }{ }^{40}\end{array}$ \\
\hline
\end{tabular}

Abbreviations: A-CHESS, Alcohol - Comprehensive Health Enhancement Support System; apps, applications; AUD, alcohol use disorder; BAC, blood alcohol concentration; LBMI-A, Location-Based Monitoring and Intervention system for Alcohol use disorders. 
the app. ${ }^{19}$ The number of risky drinking days in the past 30 days (more than four standard drinks for men or more than three standard drinks for women in a 2-hour period) was assessed via self-report during treatment at month 4 , at the end of treatment at month 8, and at a 4-month post-treatment follow-up. Patients who received A-CHESS reported significantly fewer risky drinking days during this 12 -month period ( $M=1.39$ vs 2.75, $P=0.003,95 \%$ confidence interval $0.46,2.27) .{ }^{18}$

Secondary analyses of A-CHESS data have been conducted to identify predictors of lapse so that future versions of the app can be developed to prevent relapse. In a recent study, weekly survey data from 152 participants were analyzed, and a model was developed to estimate the probability that a patient would lapse within 1 week of data collection. ${ }^{15}$ The model showed good sensitivity and specificity, and an alert feature was subsequently integrated into A-CHESS that sends a text message to patients and their counselors warning them of their risk of lapse and offering users advice on how to avoid a lapse (eg, attend a local Alcoholics Anonymous meeting) if their responses on surveys indicate the potential for a lapse.

Another version of A-CHESS, called ACHESS (Addiction Comprehensive Health Enhancement Support System), was combined with additional self-help resources and frequent ecological momentary assessment (six times per day). This 6-week intervention was evaluated in 29 adolescent alcohol and substance abusers discharged from residential care. ${ }^{26}$ Although the study did not examine treatment efficacy, it demonstrated the feasibility of using ACHESS for recovery support among adolescents, and it showed that participants were actively engaged with the intervention features of the app throughout the study. In fact, all participants accessed the intervention components at some point during the study, on average, accessing these features on $78 \%$ of study days. Notably, however, participants received compensation for completing ecological momentary assessments in the app, which may have influenced how frequently they engaged with the other features of ACHESS. Nevertheless, A-CHESS and ACHESS are promising apps for adults and teenagers in recovery for alcohol and substance use disorders.

\section{Location-Based Monitoring and Intervention system for Alcohol use disorders}

Location-Based Monitoring and Intervention system for Alcohol use disorders (LBMI-A) is another smartphone app that provides AUD patients with recovery support. ${ }^{27-31}$ App development was informed by several theoretical perspectives, including relapse prevention, ${ }^{25}$ community reinforcement, ${ }^{32}$ and motivational enhancement. ${ }^{33}$ LBMI-A consists of a series of psychoeducational modules: 1) motivational enhancement through assessment and feedback, 2) identification of high-risk drinking locations and strategies to avoid them, 3) selection of a social support network, 4) managing cravings, 5) problem-solving skills, 6) pleasurable alternatives to drinking, and 7) assertive communication and drink refusal skills. After reading or listening to each module, a related feature of the app becomes available to the user. For example, after completing the high-risk drinking location module, GPS-enabled tracking provides users with audible alerts when they approach high-risk locations. Other features include interactive coping tools, assessments, and motivational tools. ${ }^{29}$

In a recent pilot study, ${ }^{31} 60$ participants recruited from the general community who met the Diagnostic and Statistical Manual of Mental Disorders-5 diagnostic criteria for AUD were assigned to one of two intervention groups: 1) LBMI-A or 2) the Drinker's Check-up, an Internet-based motivational intervention to reduce alcohol use. ${ }^{34}$ Both interventions produced significant decreases in self-reported percent heavy drinking days and drinks per week from baseline to a 6-week follow-up, and LBMI-A also produced a significant increase in self-reported percent days abstinent. ${ }^{31}$ Although app usage declined over the course of the intervention, $71 \%$ of LBMI-A participants accessed all seven modules in the app. Thus, initial evidence suggests that LBMI-A is a feasible and efficacious app for promoting abstinence from alcohol. Notably, a commercial version of LBMI-A, called Step Away, was recently developed and is now available at the iTunes store. ${ }^{35}$ However, the efficacy of this version of the app has not yet been evaluated in the peer-reviewed literature.

\section{Promillekoll and PartyPlanner}

A study conducted in Sweden evaluated two Swedish-language smartphone apps designed to estimate blood alcohol concentration (BAC): Promillekoll (tr. "Check your BAC") and PartyPlanner. ${ }^{36}$ Promillekoll is a publically available app that was developed by the Swedish government based on the assumption that real-time feedback about estimated BAC can influence alcohol consumption. Users enter information about a beverage, and the app notifies them if consumption will result in a $\mathrm{BAC}>0.06 \%$. The app also contains various strategies to help users maintain their consumption at a rate that would prevent their BAC from reaching harmful levels.

PartyPlanner was developed by Gajecki et al, ${ }^{36}$ and, like Promillekoll, it provides real-time estimates of BAC based on user input. In addition, the app allows users to simulate 
a planned drinking occasion (eg, a party) prior to the actual event. Users can then compare estimated BAC levels predicted by the simulation to levels from real-time alcohol consumption during the event. Thus, the app was designed to increase awareness and control of drinking behavior by allowing users to compare predicted alcohol consumption to actual consumption.

The effects of Promillekoll and PartyPlanner were compared to a no-intervention control condition in a three-arm study with 1,932 university students identified as hazardous drinkers (ie, Alcohol Use Disorders Identification Test scores $\geq 8$ for men and $\geq 6$ for women). ${ }^{36}$ Past 7 -day self-reported quantity and frequency of alcohol consumption and number of bingedrinking episodes were assessed at baseline and a 7-week follow-up. Neither app produced significant decreases in any self-reported measure of alcohol consumption. Moreover, only $41 \%$ of PartyPlanner participants used the app, and only $74 \%$ of Promillekoll participants used it. ${ }^{36}$

Notably, the researchers who conducted this study emailed participants hyperlinks to the apps. Thus, researchers did not demonstrate how to use the app or install it on participants' phones. In fact, research staff never met directly with participants - all communication was conducted remotely. This procedure may have increased the social validity of the study because smartphone apps are typically downloaded and used by consumers without face-to-face instruction or supervision from experts. However, the lack of instruction and initial supervision at the beginning of the study limited some participants' exposure to the apps and may have contributed to the low levels of app engagement observed during the study.

\section{HealthCall-S}

HealthCall is an mHealth intervention designed to enhance motivational interviewing (MI $)^{33}$ and extend treatment for HIV patients with comorbid AUD. The first version of HealthCall relied on an automated interactive voice response (IVR) system that could be used with any type of telephone. ${ }^{37,38}$ Following an MI session, daily IVR calls were used to collect data on alcohol consumption over a 60 -day period. Patients met with their MI counselors at 30 and 60 days and were presented with personalized feedback graphs of their drinking behavior to facilitate counseling. In a three-group randomized controlled trial, participants who received the HealthCall-IVR intervention $(n=88)$ reported fewer drinks per drinking day $(M=3.58)$ than participants assigned to an MI-only condition $(\mathrm{n}=82 ; M=3.94)$ or an advice/education control condition $(\mathrm{n}=88 ; M=4.75, P=0.01){ }^{38}$
HealthCall was subsequently adapted for delivery via a smartphone app called HealthCall-S. ${ }^{39}$ In HealthCall-S, responses to daily questionnaires are entered via a smartphone touchpad screen. The app also allows patients to view a graph showing the number of daily drinks over the previous 7 days, and it provides an option for patients to contact their counselors when at-risk drinking is reported. A pilot study of HealthCall-S was recently conducted in 41 alcohol-dependent HIV-infected patients, ${ }^{39}$ and outcomes from these participants were compared to outcomes from 43 alcohol-dependent patients assigned to the HealthCall-IVR group in the randomized controlled trial discussed earlier. ${ }^{38}$ The number of drinks per drinking day was similar across groups, and the HealthCall-S app was used by patients on more days during the intervention (median $=85 \%$ ) than HealthCall-IVR was used $($ median $=64 \%, P<0.001) .{ }^{39}$ However, treatment efficacy remains unclear until a more rigorous empirical study can be conducted.

\section{Chimpshop}

Chimpshop ${ }^{40,41}$ is a gamified version of the Alcohol AttentionControl Training Program, ${ }^{42}$ a computerized intervention designed to reduce alcohol consumption by training users to overcome alcohol attentional bias. In Chimpshop, players can earn points by selecting healthy products and avoiding alcohol-related products in a virtual supermarket setting. The intervention is believed to reduce alcohol users' attentional bias toward alcohol-related stimuli. Although systematic evaluation of the app is incomplete, preliminary findings from a pilot study suggest that playing Chimpshop for 30 minutes per week is associated with a reduction in self-reported alcohol consumption..$^{40} \mathrm{~A}$ more rigorous efficacy study is currently underway, and Chimpshop is commercially available at Google Play and iTunes stores.

\section{Discussion}

Our literature search identified only six smartphone apps designed to treat AUD or reduce alcohol consumption that have been evaluated in the peer-reviewed scientific literature: A-CHESS, LBMI-A, Promillekoll, PartyPlanner, HealthCall-S, and Chimpshop. A-CHESS and LBMI-A primarily use cognitive-behavioral relapse prevention techniques to extend treatment and facilitate recovery among individuals with AUD. Initial evidence suggests that these apps may be efficacious in reducing alcohol consumption. ${ }^{18,31}$ However, more research on both apps is needed to replicate and extend these findings, and future studies should include objective measures of alcohol consumption. Promillekoll and PartyPlanner can be used to estimate BAC from user input regarding 
the quantity and quality of alcoholic beverages. In the study that evaluated these two interventions, the apps were not shown to reduce alcohol consumption. ${ }^{36}$ Notably, researchers emailed hyperlinks to the apps to participants, and participants did not regularly use the apps. Although research suggests that computerized alcohol interventions are more efficacious than assessment-only controls, alcohol interventions that involve some form of face-to-face contact are more efficacious than those that are delivered only electronically. ${ }^{43}$ Thus, future studies should involve more direct interaction with and supervision from research or clinic staff. Finally, two other apps, HealthCall-S and Chimpshop, are in earlier stages of evaluation. HealthCall- $\mathrm{S}^{39}$ relies primarily on self-monitoring to promote reductions in alcohol use, and Chimpshop ${ }^{40,41}$ is a game designed to reduce alcohol consumption by training users to overcome alcohol attentional bias. Initial investigations of these apps are promising, but much more rigorous empirical investigation is needed for both apps.

Although smartphone-based mHealth apps have the potential to promote reductions in alcohol consumption and extend care for individuals diagnosed with AUD, the funding, expertise, and other resources that are required to develop a smartphone app may be cost prohibitive for some researchers and practitioners. However, industry is producing alcohol reduction smartphone apps at a remarkable pace. Thus, it is important for AUD researchers to evaluate this existing technology. Crane et $\mathrm{l}^{14}$ conducted a content analysis of 61 commercially available alcohol reduction apps and found that $88 \%$ of the apps contained some, albeit, a limited number of, theoretically or empirically derived behavior change techniques. The most common behavior change techniques used by the apps were 1) facilitating self-recording (in 54\% of apps), 2) informing the user about the consequences of alcohol consumption and abstinence (in $43 \%$ of apps), and 3 ) providing feedback on performance (in $41 \%$ of apps). More research is needed to systematically evaluate commercially available alcohol reduction apps that use these and other behavior change techniques.

Whether alcohol-related smartphone apps are the product of research or industry, their systematic evaluation and potential efficacy could be enhanced by measuring physiological indices of alcohol craving and consumption. Thus, several researchers have worked to develop smartphone-compatible hardware and software to assess objective measures of consumption and physiological indices associated with craving. For example, Baskett et $\mathrm{al}^{44}$ recently published a technical report on a multi-sensor system designed to assess alcohol craving and mood dysregulation through accelerometry, skin temperature, electro dermal activity, heart rate, respira- tion, and electrocardiography. This technology is similar to "iHeal", a system involving artificial intelligence, biophysical monitoring, and smartphone technology that is being developed to detect illicit drug craving. ${ }^{45}$ In addition, research ${ }^{46}$ and industry (eg, BACtrack ${ }^{\circledR}$ Mobile Pro) have developed alcohol breathalyzers that can communicate directly with smartphone apps. This technology could be particularly useful when combined with mHealth treatment approaches that require objective assessment of alcohol consumption, such as incentive-based treatment. ${ }^{12}$

\section{Conclusion}

While the commercial production of alcohol-related apps is proliferating, the results of this review suggest that systematic evaluations of smartphone apps to promote reductions in alcohol consumption or treat AUD are scarce. Much more research is needed to determine whether mHealth apps can be used to treat AUD or promote abstinence from alcohol. Nevertheless, the advantages of this technology are clear. Smartphone apps have the potential to monitor alcohol use and craving in the natural environment and deliver tailored treatment to users when they need it most. In addition, the dissemination potential of mHealth apps continues to grow as the rate of smartphone ownership increases. Thus, this technology can be used not only by patients receiving clinical care for AUD but also by hazardous drinkers and undiagnosed individuals who are not accessing the health care system.

\section{Acknowledgments}

Preparation of this report was supported in part by National Institutes of Health grants R01-DA027615, R01-DA13444, P50-DA09241, P60-AA03510, R01-HD075630, R01AA021446, and R01-AA023502.

\section{Disclosure}

The authors report no conflicts of interest in this work.

\section{References}

1. World Health Organization. Global Status Report on Alcohol and Health. Geneva, Switzerland: WHO Press; 2014. Available from: http:// apps.who.int/iris/bitstream/10665/112736/1/9789240692763_eng.pdf. Accessed July 13, 2015.

2. American Psychiatric Association. Diagnostic and Statistical Manual of Mental Disorders. 5th ed. Washington, DC: American Psychiatric Association; 2013.

3. Grant BF, Goldstein RB, Saha TD, et al. Epidemiology of DSM-5 alcohol use disorder: results from the national epidemiologic survey on alcohol and related conditions III. JAMA Psychiatry. 2015;72(8):757-766.

4. White WL, Boyle M, Loveland D. Alcoholism/addiction as a chronic disease. Alcohol Treat Q. 2002;20(3-4):107-129. 
5. Saunders JB, Lee NK. Hazardous alcohol use: its delineation as a subthreshold disorder, and approaches to its diagnosis and management. Compr Psychiatry. 2000;41(2 Suppl 1):95-103.

6. GSMA Intelligence. The mobile economy. 2015. Available from: http:// gsmamobileeconomy.com/global/GSMA_Global_Mobile_Economy_ Report_2015.pdf. Accessed July 13, 2015.

7. Pew Research Center. Cell phone and smartphone ownership demographics. 2014. Available from: http://www.pewinternet.org/datatrend/mobile/cell-phone-and-smartphone-ownership-demographics/. Accessed July 13, 2015.

8. Quanbeck A, Chih M, Isham A, Johnson R, Gustafson D. Mobile delivery of treatment for alcohol use disorders. Alcohol Res. 2014;36(1):111-112.

9. Agyapong VI, Ahern S, McLoughlin DM, Farren CK. Supportive text messaging for depression and comorbid alcohol use disorder: singleblind randomised trial. J Affect Disord. 2012;141(2-3):168-176.

10. Agyapong VI, McLoughlin DM, Farren CK. Six-months outcomes of a randomised trial of supportive text messaging for depression and comorbid alcohol use disorder. J Affect Disord. 2013;151(1):100-104.

11. Keoleian V, Polcin D, Galloway GP. Text messaging for addiction: a review. J Psychoactive Drugs. 2015;47(2):158-176.

12. Alessi SM, Petry NM. A randomized study of cellphone technology to reinforce alcohol abstinence in the natural environment. Addiction. 2013;108(5):900-909.

13. Smith A. US smartphone use in 2015. 2015. Available from: http://www. pewinternet.org/2015/04/01/us-smartphone-use-in-2015/. Accessed July 13, 2015.

14. Crane D, Garnett C, Brown J, West R, Michie S. Behavior change techniques in popular alcohol reduction apps: content analysis. J Med Internet Res. 2015;17(5):e118.

15. Chih MY, Patton T, McTavish FM, et al. Predictive modeling of addiction lapses in a mobile health application. $J$ Subst Abuse Treat. 2014;46(1):29-35.

16. Gustafson DH, Boyle MG, Shaw BR, et al. An e-health solution for people with alcohol problems. Alcohol Res Health. 2011;33(4):327-337.

17. Gustafson DH, Shaw BR, Isham A, Baker T, Boyle MG, Levy M Explicating an evidence-based, theoretically informed, mobile technology-based system to improve outcomes for people in recovery for alcohol dependence. Subst Use Misuse. 2011;46(1):96-111.

18. Gustafson DH, McTavish FM, Chih MY, et al. A smartphone application to support recovery from alcoholism a randomized clinical trial; 24671165. JAMA Psychiatry. 2014;71(5):566-572.

19. McTavish FM, Chih MY, Shah D, Gustafson DH. How patients recovering from alcoholism use a smartphone intervention. J Dual Diagn. 2012;8(4):294-304.

20. Gustafson DH, McTavish FM, Stengle W, et al. Use and impact of eHealth system by low-income women with breast cancer. $J$ Health Commun. 2005;10(Suppl 1):195-218.

21. Gustafson DH, DuBenske LL, Namkoong K, et al. An eHealth system supporting palliative care for patients with non-small cell lung cancer: a randomized trial. Cancer. 2013;119(9):1744-1751.

22. Gustafson D, Wise M, Bhattacharya A, et al. The effects of combining web-based eHealth with telephone nurse case management for pediatric asthma control: a randomized controlled trial. $J$ Med Internet Res. 2012;14(4):e101

23. Temesgen Z, Knappe-Langworthy JE, St Marie MM, Smith BA, Dierkhising RA. Comprehensive health enhancement support system (CHESS) for people with HIV infection. AIDS Behav. 2006; 10(1):35-40

24. Ryan RM, Deci EL. Self-determination theory and the facilitation of intrinsic motivation, social development, and well-being. Am Psychol. 2000;55(1):68-78

25. Marlatt GA, George WH. Relapse prevention: introduction and overview of the model. Br J Addict. 1984;79(3):261-273.

26. Dennis ML, Scott CK, Funk RR, Nicholson L. A pilot study to examine the feasibility and potential effectiveness of using smartphones to provide recovery support for adolescents. Subst Abus. 2015;36(4): 486-492.
27. Dulin PL, Gonzalez VM, King DK, Giroux D, Bacon S. Development of a smartphone-based, self-administered intervention system for alcohol use disorders. Alcohol Treat Q. 2013;31(3):321-336.

28. Dulin PL, Gonzalez VM, King DK, Giroux D, Bacon S. Smartphonebased, self-administered intervention system for alcohol use disorders: theory and empirical evidence basis. Alcohol Treat Q. 2013;31(3). doi:10.1080/07347324.2013.800425.

29. Dulin PL, Gonzalez VM, Campbell K. Results of a pilot test of a self-administered smartphone-based treatment system for alcohol use disorders: usability and early outcomes. Subst Abus. 2014;35(2): $168-175$.

30. Giroux D, Bacon S, King DK, Dulin P, Gonzalez V. Examining perceptions of a smartphone-based intervention system for alcohol use disorders. Telemed J E Health. 2014;20(10):923-929.

31. Gonzalez VM, Dulin PL. Comparison of a smartphone app for alcohol use disorders with an internet-based intervention plus bibliotherapy: a pilot study. J Consult Clin Psychol. 2015;83(2):335-345.

32. Smith JE, Meyers RJ. Motivating Substance Abusers to Enter Treatment: Working with Family Members. New York: Guilford Press; 2008.

33. Miller WR, Rollnick S. Motivational Interviewing: Helping People Change. 3rd ed. New York: Guilford Press; 2012.

34. Hester RK, Squires DD, Delaney HD. The drinker's check-up: 12-month outcomes of a controlled clinical trial of a stand-alone software program for problem drinkers. $J$ Subst Abuse Treat. 2005;28(2):159-169.

35. Dulin P, Gonzalez V, Fitterling J. Development of a stand-alone, smartphone-based system for changing drinking. The Addictions Newsletter. The American Psychological Association, Division 50; Spring 2014:12-14.

36. Gajecki M, Berman AH, Sinadinovic K, Rosendahl I, Andersson C. Mobile phone brief intervention applications for risky alcohol use among university students: a randomized controlled study. Addict Sci Clin Pract. 2014;9:11. doi:10.1186/1940-0640-9-11.

37. Aharonovich E, Hatzenbuehler ML, Johnston B, et al. A low-cost, sustainable intervention for drinking reduction in the HIV primary care setting. AIDS Care. 2006;18(6):561-568.

38. Hasin DS, Aharonovich E, O'Leary A, et al. Reducing heavy drinking in HIV primary care: a randomized trial of brief intervention, with and without technological enhancement. Addiction. 2013;108(7): 1230-1240.

39. Hasin DS, Aharonovich E, Greenstein E. HealthCall for the smartphone: technology enhancement of brief intervention in HIV alcohol dependent patients. Addict Sci Clin Pract. 2014;9:5. doi:10.1186/19400640-9-5.

40. Cox WM, Fadardi JS, Intriligator JM, Klinger E. Attentional bias modification for addictive behaviors: clinical implications. CNS Spectr. 2014;19(3):215-224.

41. Cox M, Intriligator J, Hillier C. Chimpshop and alcohol reduction using technology to change behaviour. Perspect Public Health. 2015;135(3):126-127.

42. Fadardi JS, Cox WM. Reversing the sequence: reducing alcohol consumption by overcoming alcohol attentional bias. Drug Alcohol Depend. 2009;101(3):137-145.

43. Carey KB, Scott-Sheldon LA, Elliott JC, Garey L, Carey MP. Faceto-face versus computer-delivered alcohol interventions for college drinkers: a meta-analytic review, 1998 to 2010. Clin Psychol Rev. 2012;32(8):690-703.

44. Baskett P, Shang Y, Patterson MV, Trull T. Towards a system for bodyarea sensing and detection of alcohol craving and mood dysregulation. In: 2013 IEEE 10th Consumer Communications and Networking Conference; 2013:875-876.

45. Boyer EW, Fletcher R, Fay RJ, Smelson D, Ziedonis D, Picard RW. Preliminary efforts directed toward the detection of craving of illicit substances: the iHeal project. J Med Toxicol. 2012;8(1):5-9.

46. Yang S, Lee J, Sul S, Kim M, Choi J. Ear-jack port based one-way analogue communication for smart devices. J Mech Sci Technol. 2014; 28(8):3147-3152. 
Advanced Health Care Technologies

Dovepress

\section{Publish your work in this journal}

Advanced Health Care Technologies is an international, peer reviewed, open access journal that provides a unique forum for articles on: point-of-care, health care diagnostics and treatment, bioengineering, biotechnology, biosensing, electronics, clinical/medical science, chemical engineering, materials science, regenerative medicine, micro-/

nano-technologies, and methods and applications for nanoscience and nanotechnology. The manuscript management system is completely online and includes a very quick and fair peer review system, which is all easy to use. Visit http://www.dovepress.com/testimonials.php to read real quotes from published authors.

Submit your manuscript here: http://www.dovepress.com/advanced-health-care-technologies-journal 